\title{
Inverse-problem approach to designing photonic crystals for cavity QED experiments
}

\author{
J. M. Geremia,* Jon Williams, and Hideo Mabuchi \\ Institute for Quantum Information, Mail Code 12-33, California Institute of Technology, Pasadena, California 91125
}

(Received 13 June 2002; published 16 December 2002)

\begin{abstract}
Photonic band gap (PBG) materials are attractive for cavity QED experiments because they provide extremely small mode volumes and are monolithic, integratable structures. As such, PBG cavities are a promising alternative to Fabry-Perot resonators. However, the cavity requirements imposed by QED experiments, such as the need for high $Q$ (low cavity damping) and small mode volumes, present significant design challenges for photonic band gap materials. Here, we pose the PBG design problem as a mathematical inversion and provide an analytical solution for a two-dimensional (2D) crystal. We then address a planar (2D crystal with finite thickness) structure using numerical techniques.
\end{abstract}

DOI: 10.1103/PhysRevE.66.066606

PACS number(s): 42.70.Qs, 32.80.Pj, 12.20.Fv

\section{INTRODUCTION}

Engineering new materials to meet specific design objectives often begins by trial and error. New structures are repeatedly proposed and characterized, and the results from each iteration are used to further refine the design. This process continues until an apparent optimum is achieved-that is, when the incremental modifications stop leading to improvements.

However, from an implementation standpoint, trial and error is inefficient and costly, even when the process can be computationally simulated. Conceptually, trial and error provides little information about the quality of the optimum. This is because the design space is often too large to permit an exhaustive search. Therefore, it is common to fall back upon physical intuition (that might not apply to the new material) to guide the engineering process. Of course, this is not to say that design by trial is ineffective, only that it lacks a certain degree of rigor.

Going beyond incremental design procedures requires an algorithmic, rather than intuitive, process. In many cases, posing the design problem as a mathematical inversion [1,2] can provide an assessment of the resulting optimum. Ideally, algorithmic searches might uncover alternatives in the design space that physical intuition failed to recognize. However, such an unconstrained optimum structure might prove too difficult to manufacture, in which case, the inversion optimization can be restricted to account for limitations in the fabrication capabilities. Both alternatives are beneficial. The unconstrained inversion provides an indication of the absolute optimal performance of the material, while the constrained inversion produces the best structure that can actually be constructed.

Engineering the optical properties of photonic band gap (PBG) structures [3-6] is a process that can benefit from inversion techniques. Here, the objective is to tailor the electromagnetic modes of the crystal by adjusting its spatially dependent dielectric function. Specifically, by introducing a defect into an otherwise periodic crystal, it is possible to

\footnotetext{
*Electronic address: jgeremia@Caltech.EDU; URL:http:// minty.caltech.edu/MabuchiLab
}

produce localized electromagnetic fields [7]. Both the spatial and temporal properties of these cavity modes are affected by the geometry of the defect.

The ability to localize light fields has made photonic crystals attractive for experiments in cavity QED $[8,9]$ and the quantum information sciences $[10,11]$. PBG cavities offer a number of advantages that make them an attractive alternative to Fabry-Perot resonators. Most notably, a small cavity mode volume is an important factor in achieving the strong coupling limit between the trapped atoms and the cavity light field. Photonic crystal cavities are capable of mode volumes of the order of the cubic wavelength of the light $[9,12]$.

Modern lithographic techniques should enable the integration of PBG structures with micronscale magnetic traps for neutral atoms [13]. Atom trapping experiments, however, pose nontrivial design challenges for photonic crystals. Practical concerns in cavity QED experiments, such as atom delivery and confinement, strongly suggest using planar photonic crystals [7,12,14-19] (two-dimensional lattices with finite thickness) rather than full 3D materials. However, twodimensional (2D) PBG crystals only provide incomplete, quasi-3D light trapping. While well confined within the lattice plane, the cavity field can decay in the out-of-plane direction $[8,20,21]$ by coupling to the radiated modes. Radiation loss should generally be the most significant decay mechanism in planar photonic cavities [8]. Therefore, maximizing the cavity quality factor $Q=\Delta \omega_{j} / \omega_{j}$ requires that this radiation loss be minimized. Simultaneously, cavity QED experiments require that the cavity mode function have high relative field strengths in vacuum regions (as opposed to inside the semiconductor) which are accessible to the trapped atoms. Otherwise, the atomic system will not couple strongly to the cavity field. Additionally, these criteria must be met without sacrificing mode volume, i.e., by delocalizing the defect field.

There has recently been considerable progress toward PBG cavities that display the necessary properties for QED. Numerical design work performed by Scherer and coworkers $[8,22]$ has identified planar photonic crystals $[23,18,21]$ with mode volumes on the order of the cubic wavelength of the light and cavity $Q$ factors $\sim 10^{4}$, which is sufficient for strong coupling. However, with these structures, it is difficult to know if they are true optima. Further- 
more, if they are not optimal, it is unclear how to further improve them, i.e., whether small modifications or large changes in the crystal would be needed.

This paper poses the photonic crystal design process as an inverse problem in order to provide an algorithmic optimization. That is, we represent the defect dielectric function as resulting from the design requirements, rather than proposing a defect geometry and then characterizing the crystal to observe its properties. To the best of our knowledge, this paper represents the first attempt to treat photonic band gap materials in such a manner. We demonstrate that mathematical inversion leads to photonic crystal structures not previously suggested by intuitive or trial and error design techniques, and we also illustrate how fabrication-imposed constraints can be placed on the inversion optimization.

When solving inverse problems, there are two possible directions to follow. The first is to analytically treat a simplified model that captures the relevant properties of the actual problem. Analytic solutions are rarely possible for the structures of arbitrary (realistic) complexity; however, they provide a closed mathematical description, and hopefully a better understanding, of the design process. In Sec. III, we present analytic results for a pure (i.e., infinitely thick) twodimensional photonic crystal. The second class of inversion algorithms utilize numerical methods to treat more realistic descriptions of the underlying physics. However, in exchange for the more realistic model, it can be difficult to find global extrema in the design space. In Sec. IV, we employ numerical methods to treat a planar photonic crystal membrane.

\section{CAVITY DESIGN AS AN INVERSE PROBLEM}

The relationship between the spatially dependent dielectric function $\kappa(\mathbf{r})$ [24], and the properties of interest, such as mode volume and $Q$, is a composition of two individual components. First, the electric and magnetic fields are related to the reciprocal dielectric function $\eta(\mathbf{r})=1 / \kappa(\mathbf{r})$, through the Maxwell curl operator,

$$
\boldsymbol{\nabla} \times \eta(\mathbf{r}) \boldsymbol{\nabla} \times \mathbf{H}_{j}(\mathbf{r})=\frac{\omega_{j}^{2}}{c^{2}} \mathbf{H}_{j}(\mathbf{r}),
$$

where $\mathbf{H}_{j}(\mathbf{r})$ is the magnetic field for the mode $j$, with frequency $\omega_{j}$. It is often most convenient to work with $\mathbf{H}$ because the resulting Maxwell equation is Hermitian. This provides no difficulty because the electric field

$$
\mathbf{E}_{j}(\mathbf{r})=i \boldsymbol{\nabla} \times \mathbf{H}_{j}(\mathbf{r}) / \omega_{j} \epsilon_{0} \kappa(\mathbf{r})
$$

can always be found from the magnetic field.

The second relationship then connects the photonic crystal's electromagnetic modes to its physical characteristics. In some cases, the property of interest can be directly expressed, such as for the QED mode volume [25]

$$
\mathcal{V}_{j}=\int\left|\psi_{j}(\mathbf{r})\right|^{2} d \mathbf{r}
$$

Here, $j$ is the mode index and $\psi(\mathbf{r})$ is a normalized spatial wave function,

$$
\mathbf{H}_{j}(\mathbf{r})=\mathbf{H}_{0, j} \psi_{j}(\mathbf{r}),
$$

where $\mathbf{H}_{0, j}$ is chosen such that $\psi_{j}$ is max-one normalized, i.e., $|\psi(\mathbf{r})|_{\max }=1$ [26]. It is also possible to directly express the magnitude of the field at the location $\mathbf{r}_{a}$ of the trapped atom,

$$
I_{j}=\left|\mathbf{H}_{j}\left(\mathbf{r}_{a}=0\right)\right|^{2},
$$

where $\mathbf{r}_{a}=0$ can always be achieved by a suitable choice of axes.

For other characteristics, such as the cavity $Q$, it may be too difficult, or inappropriate, to analytically express the property as a function of the electromagnetic modes. For the cavity $Q$, it is more convenient to work with some other measure, $(L)$,

$$
Q_{j} \sim L_{j}\left[\mathbf{H}_{j}(\mathbf{r})\right]
$$

that acts as a proxy for an actual calculation of the cavity $Q$. This measure must display the property that maximizing it simultaneously maximizes $Q$ (this will be discussed in greater detail in Sec. III C).

\section{Inversion cost functional}

In all these cases, the fundamental relationships that connect the properties of the electromagnetic modes to the reciprocal dielectric function $\eta(\mathbf{r})$ remain implicit,

$$
Q_{j} \rightarrow Q_{j}[\eta(\mathbf{r})], \quad \mathcal{V}_{j} \rightarrow \mathcal{V}_{j}[\eta(\mathbf{r})], \quad I_{j} \rightarrow I_{j}[\eta(\mathbf{r})] .
$$

Here, the notation $[\cdots]$ represents the fact that the quantities are complicated functionals of their input. This is because Eq. (1) has been buried inside them. Therefore, evaluating the functionals for any given crystal entails solving Maxwell's equations and then computing the property from the resulting modes.

Nonetheless, with these implicit functionals in hand, it is possible to formally state the inverse problem by defining a cost functional

$$
\mathcal{J}[\eta(\mathbf{r})]=Q_{m}[\eta(\mathbf{r})]+\beta_{I} I_{m}[\eta(\mathbf{r})]-\beta_{\mathcal{V}} \mathcal{V}_{m}[\eta(\mathbf{r})]
$$

evaluated for the appropriate cavity mode $j=m$ [27]. $\beta_{I}$ and $\beta_{\mathcal{V}}$ are scalars that balance the relative importance of the various terms in the cost. Solving the inverse problem is accomplished by optimizing $\mathcal{J}$,

$$
\eta^{*}=\max _{\eta(\mathbf{r})} \mathcal{J}[\eta(\mathbf{r})]
$$

over the possible structures (indexed by their dielectric function).

As with any inversion that is performed by optimization, there are many possible choices for the cost functional. However, the essential features of the cost are that it should capture all of the relevant physical design objectives being optimized, while remaining mathematically well suited for 
optimization. It can be seen that Eq. (8) includes terms for the mode volume, the cavity $Q$, and the peak field intensity. So, the first requirement has been met. The specific formulations of the individual terms in Eq. (8) depend on the structure of the photonic crystal and the techniques being used to optimize it. These points will be demonstrated in the following two sections.

Of course, Eq. (8) is deceptively simple-all the details of solving the inverse problem have been relegated to the $[\cdots]$ notation. However, there is an important advantage to such an abstraction. Equation (8) provides a language for describing the photonic crystal structure in terms of the design objective. It also provides a description of the inversion that is independent of the particular method used to solve Maxwell's equations. $\mathcal{J}[\eta(\mathbf{r})]$ is easily generalized to design objectives other than the cavity $Q$ and mode volume, and it applies to 2D, planar, and full 3D materials.

In the following two sections, Eqs. (8) and (9) are solved for specific examples. First, an analytic approach is used to treat an infinitely thick two-dimensional crystal. In this case, handling the $[\cdots]$ calls for the majority of the effort. But once this is accomplished, the optimization is relatively straightforward. The second example incorporates numerical methods to treat a planar 2D crystal. Here, there is no struggle with the notation-we just write a computer program to compute the mode volume and cavity $Q$. However, the optimization is complicated by the possibility of local minima.

\section{ANALYTICAL INVERSION}

In this section, the photonic crystal inversion is analytically performed for a two-dimensional structure. The 2D problem is motivated by the fact that the planar and $2 \mathrm{D}$ structures share many similarities. Treating the 2D crystal allows a detailed mathematical inversion and can provide insight into how to also optimize a planar crystal.

The general inversion strategy is to solve a variational problem by expanding the cavity field in the bulk crystal electromagnetic modes. It is therefore necessary to select a bulk 2D lattice with a band gap surrounding the desired cavity resonance frequency (such as an hexagonal array of holes with a suitable lattice constant) prior to the inversion. Once the electromagnetic modes of the bulk structure are determined, it is possible to optimize the cavity $Q$, field intensity at $\mathbf{r}_{a}$, and mode volume over the bulk mode expansion coefficients. This optimization stage does not directly involve the defect dielectric function- it identifies the optimal cavity field that can be produced using the bulk crystal modes as a basis. Once these optimal expansion coefficients are identified, the defect that produces the optimal field is extracted by inverting the Maxwell curl equation (1).

\section{A. Bulk crystal modes}

Methods for solving the Maxwell equations for a twodimensional photonic crystal are well established $[6,28]$. However, it is useful to briefly review the plane wave expansion method in order to provide sufficient context for solving the inverse problem.
The two-dimensional photonic crystal consists of a bulk medium with an index of refraction $n_{b}$. It is laced with a lattice of infinitely deep cylindrical holes with radius $r_{h}$ and index of refraction $n_{h}$. This lattice is represented by the real-space vectors $\left\{\mathbf{R}_{n}\right\}$ which point from the origin to the centers of the cylinders. All of the real-space lattice vectors lie in a plane.

Since the inverse dielectric function $\eta_{0}(\mathbf{r})$ for the bulk crystal is periodic, it is convenient to work with its Fourier transform,

$$
\eta_{0}(\mathbf{r})=\sum_{\mathbf{G}} \eta_{\mathbf{G}} e^{i \mathbf{G} \cdot \mathbf{r}}
$$

where the reciprocal lattice vectors $\mathbf{G}$ satisfy $\mathbf{G} \cdot \mathbf{R}_{n}=2 l \pi$, $l=1,2, \ldots$. Physically, each reciprocal lattice vector is the wave vector of a plane wave that shares the periodicity of the real-space lattice.

As with the dielectric function, the bulk crystal electromagnetic modes are periodic in the lattice. In accordance with Bloch's theorem [29], the bulk crystal electromagnetic modes can also be expanded in the reciprocal lattice vectors,

$$
\mathbf{H}_{n, \mathbf{q}}(\mathbf{r})=\sum_{\lambda} \hat{\mathbf{e}}_{\lambda} \sum_{\mathbf{G}} h_{n, \mathbf{q}+\mathbf{G}} e^{i(\mathbf{q}+\mathbf{G}) \cdot \mathbf{r}},
$$

where the mode is labeled by its wave vector $\mathbf{q}[30]$ and band index $n$. The $\hat{\mathbf{e}}_{\lambda}$ are orthogonal polarization vectors and the $h_{n, \mathbf{q}+\mathbf{G}}$ are the plane wave expansion coefficients that produce the mode.

Calculating the bulk crystal modes is accomplished by solving the Maxwell equation (1), using the form in Eq. (11). This leads to wave equations for the two possible polarizations,

$$
\sum_{\mathbf{G}^{\prime}} \eta_{\mathbf{G}^{-}-\mathbf{G}^{\prime}}(\mathbf{q}+\mathbf{G}) \cdot\left(\mathbf{q}+\mathbf{G}^{\prime}\right) h_{n, \mathbf{q}+\mathbf{G}^{\prime}}=\frac{\omega_{n, \mathbf{q}}^{2}}{c^{2}} h_{n, \mathbf{q}+\mathbf{G}}
$$

for TE modes, and

$$
\sum_{\mathbf{G}^{\prime}} \eta_{\mathbf{G}^{-}-\mathbf{G}^{\prime}}\left|\mathbf{q}+\mathbf{G} \| \mathbf{q}+\mathbf{G}^{\prime}\right| h_{n, \mathbf{q}+\mathbf{G}^{\prime}}=\frac{\omega_{n, \mathbf{q}}^{2}}{c^{2}} h_{n, \mathbf{q}+\mathbf{G}}
$$

for TM modes. Since the polarizations uncouple for a pure two-dimensional crystals, it is possible to work with them independently. For the remainder of this section, we utilize TE modes; however, the same inversion technique applies equally well to TM modes.

\section{B. Defect crystal modes}

The cavity mode $m$ can be expanded in the bulk modes $\mathbf{H}_{n, \mathbf{q}}(\mathbf{r})$ using wave vectors that are confined to the first Brillouin zone (see, for example, Ref. [29]),

$$
\mathbf{H}_{m}(\mathbf{r})=\sum_{n} \sum_{\mathbf{q} \in B Z} a_{n, \mathbf{q}}^{(m)} \mathbf{H}_{n, \mathbf{q}}(\mathbf{r}),
$$


of the two-dimensional lattice. This superposition is the reason for working with the magnetic, rather than electric, fields. Since Eq. (1) is Hermitian, the bulk modes are complete.

The photonic crystal cavity can be described by introducing an additional defect term $\delta \eta(\mathbf{r})$ into the reciprocal dielectric function

$$
\eta(\mathbf{r})=\eta_{0}(\mathbf{r})+\delta \eta(\mathbf{r})
$$

whose Fourier transform is given by

$$
\delta \eta(\mathbf{r})=\int_{\mathbf{k}} d \mathbf{k} \delta \eta(\mathbf{k}) e^{i \mathbf{k} \cdot \mathbf{r}} \approx \sum_{\mathbf{k}} \delta \eta_{\mathbf{k}} e^{i \mathbf{k} \cdot \mathbf{r}} .
$$

However, unlike the bulk lattice, the cavity is not periodic, so the Fourier expansion must run over all wave vectors $\mathbf{k}$. In practice, the integral is generally approximated by a discrete sum that is then truncated to allow computation.

The coefficients $a_{n, \mathbf{k}}^{(m)}$ are calculated by substituting Eqs. (14) and (15) into the Maxwell equation (1). This produces the matrix eigenvalue equation $[6,28]$

$$
\sum_{n^{\prime}, \mathbf{q}^{\prime}} A_{n, \mathbf{q} ; n^{\prime}, \mathbf{q}^{\prime}}^{(m)} a_{n^{\prime}, \mathbf{q}^{\prime}}^{(m)}=\frac{\omega_{m}^{2}}{c^{2}} a_{n, \mathbf{q}}^{(m)},
$$

where

$$
\begin{aligned}
A_{n, \mathbf{q} ; n^{\prime}, \mathbf{q}^{\prime}}^{(m)}= & \sum_{\mathbf{G}, \mathbf{G}^{\prime}} h_{n, \mathbf{q}+\mathbf{G}}^{*} \delta \eta_{\mathbf{q}+\mathbf{G}-\mathbf{q}^{\prime}-\mathbf{G}^{\prime}} h_{n^{\prime}, \mathbf{q}^{\prime}+\mathbf{G}^{\prime}} \\
& \times(\mathbf{q}+\mathbf{G}) \cdot\left(\mathbf{q}^{\prime}+\mathbf{G}^{\prime}\right)+\delta_{n, n^{\prime}} \delta_{\mathbf{q}, \mathbf{q}^{\prime}} \frac{\omega_{n, \mathbf{q}}}{c^{2}} .
\end{aligned}
$$

Here, it can be seen that the point defect couples all of the bulk crystal modes. However, the Fourier coefficients $\delta \eta_{\mathbf{k}}$ fall off quickly as the magnitude of the wave vector increases.

\section{Cavity mode optimization}

To optimize the inversion cost functional $\mathcal{J}$, it is necessary to express Eq. (8) in terms of the defect crystal modes by utilizing the expansion (14). When performing an actual inversion calculation, this is the point when it would be necessary to select a bulk photonic crystal geometry, such as a hexagonal lattice. Once this has been done, the plane wave representations of the optimization basis functions, $\mathbf{H}_{n, \mathbf{q}}(\mathbf{r})$, can be computed.

Another important point is that the optimization is performed over $\delta \eta(\mathbf{r})$, not the bulk lattice function $\eta_{0}(\mathbf{r})$. In principle, this does not restrict the optimization in any way. The distinction between $\eta_{0}(\mathbf{r})$ and $\delta \eta(\mathbf{r})$ is not perturbative, so there is no requirement on the relative magnitudes of the two functions. However, in practice, it can be practical to restrict the structure of the defect (for example, to enforce radial symmetry) in order to limit the number of plane waves (or equivalently, the number of reciprocal lattice vectors) needed for Eq. (11) to converge.

\section{Cavity mode volume and intensity}

Expressing the cavity mode volume in terms of the basis function coefficients is straightforward,

$$
\mathcal{V}_{m}=\sum_{n^{\prime}, \mathbf{q}^{\prime}} \sum_{n, \mathbf{q}} a_{n^{\prime}, \mathbf{q}^{\prime}}^{(m) *} a_{n, \mathbf{q}}^{(m)}\left\langle\psi_{n^{\prime}, \mathbf{q}^{\prime}}(\mathbf{r}) \mid \psi_{n, \mathbf{q}}(\mathbf{r})\right\rangle+\text { c.c. },
$$

as is taking derivatives with respect to the coefficients,

$$
\frac{d \mathcal{V}_{m}}{d a_{j}}=\sum_{n, \mathbf{q}} a_{n, \mathbf{q}}^{(m)}\left\langle\psi_{j}(\mathbf{r}) \mid \psi_{n, \mathbf{q}}(\mathbf{r})\right\rangle
$$

Here, the spatial functions $\psi_{j}(\mathbf{r})$ are the quantities defined in Eq. (4), and the inner product $\left\langle\psi_{n^{\prime}, \mathbf{q}^{\prime}} \mid \psi_{n, \mathbf{q}}\right\rangle$ denotes integration over the real-space domain in which the mode volume is to be minimized.

Similarly, the field intensity at the location of the trapped atom is given by

$$
I_{m}=\sum_{n^{\prime}, \mathbf{q}^{\prime}} \sum_{n, \mathbf{q}} a_{n^{\prime}, \mathbf{q}^{\prime}}^{(m) *} a_{n, \mathbf{q}}^{(m)} \mathbf{H}_{n^{\prime}, \mathbf{q}^{\prime}}^{*}(0) \mathbf{H}_{n, \mathbf{q}}(0)
$$

and the necessary derivatives with respect to the expansion coefficients are also straightforward to find. When performing an inversion calculation, both the mode volume and intensity functions can be further expanded in terms of the reciprocal lattice vector plane waves. Doing so leads to algebraic expressions in the coefficients $h_{n, \mathbf{q}+\mathbf{G}}$ from Eq. (11).

\section{Cavity $Q$ factor}

It is not as clear how to represent the cavity $Q$ in terms of the basis functions. The two-dimensional lattice is infinitely deep and therefore does not permit any radiated modes. However, this does not prevent us from minimizing features of the two-dimensional lattice that would promote out-ofplane loss were the structure a planar crystal. In other words, we wish to find the two-dimensional structure of a planar photonic crystal that minimizes the out-of-plane loss by considering features computed for a pure 2D structure. Since the cavity $Q$ cannot be directly computed, it is necessary to define an auxiliary measure of field decay that applies to the two-dimensional lattice.

The essential requirement of the auxiliary measure is that optimizing it simultaneously maximizes the cavity $Q$ for a planar structure (where radiation loss can occur). It is possible to identify such a measure by considering the physical nature of radiative field decay in a planar photonic crystal. Out-of-plane loss is the result of guided crystal modes coupling to free-space modes [8], and frequency-wave-vector pairs in free space must lie within the light cone. Therefore, bulk crystal modes with frequency-wave-vector pairs that lie below the light line should not couple to free space because they undergo total internal reflection [22].

Minimizing the contributions from bulk modes $\mathbf{H}_{n, \mathbf{q}}(\mathbf{r})$ which lie above the light line reduces radiative cavity decay. We chose to adopt the following auxiliary function: 


$$
L=\sum_{n^{\prime}, \mathbf{q}^{\prime}} \sum_{n, \mathbf{q}} a_{n^{\prime}, \mathbf{q}^{\prime}}^{(m) *} a_{n, \mathbf{q}}^{(m)} \frac{\omega_{n^{\prime}, \mathbf{q}^{\prime}} \omega_{n, \mathbf{q}}}{q q^{\prime}}
$$

where $q_{j}$ is the magnitude of its corresponding wave vector, $q_{j}=\left\|\mathbf{q}_{j}\right\|$.

Of course, since a 2D crystal does not support any unguided modes, Eq. (22) does not provide a numerical value for the cavity $Q$. It is a measure that for a $2 \mathrm{D}$ crystal is expected to behave similarly to the actual $Q$ of a planar photonic crystal with the same two-dimensional structure. This naturally raises the question of how well Eq. (22) mimics the features of a planar crystal compared to other choices for the auxiliary function. The primary motivation for using this definition of the auxiliary measure is that it has a distinct physical interpretation, while simultaneously producing an inversion cost functional that can be analytically optimized. There is substantial evidence [8] that minimizing the contribution of $2 \mathrm{D}$ wave vectors above the light line improves the cavity $Q$ of a planar crystal. Actually, Eq. (22) is a strict definition since symmetry considerations of the guided crystal modes will prevent many modes above the light line from coupling to free space. From a design perspective, it is attractive to work with a conservative measure.

\section{Analytic optimization}

The photonic cavity design characteristics, [Eqs. (19)(22)], can be substituted for their respective terms in the cost functional $\mathcal{J}[\delta \eta]$. Setting the derivatives of the design properties with respect to the expansion coefficients equal to zero produces a linear variational problem. In order to ensure that the mode functions remain properly normalized, it is convenient to impose the constraint $1-\Sigma\left|a_{n, \mathbf{q}}\right|^{2}=0$ as a Lagrange multiplier. Maximizing the resulting Lagrangian leads to a matrix eigenvalue problem,

$$
\begin{aligned}
\sum_{n^{\prime}, \mathbf{q}^{\prime}} & {\left[\frac{\omega_{n^{\prime}, \mathbf{q}^{\prime}} \omega_{n, \mathbf{q}}}{q q^{\prime}}+\beta_{I} H_{n^{\prime}, \mathbf{q}^{\prime}}^{*}(0) H_{n, \mathbf{q}}(0)\right.} \\
& \left.-\beta_{\mathcal{V}}\left\langle\psi_{n^{\prime}, \mathbf{q}^{\prime}} \mid \psi_{n, \mathbf{q}}\right\rangle\right] a_{n^{\prime}, \mathbf{q}^{\prime}}^{(m)}=\Lambda a_{n, \mathbf{q}},
\end{aligned}
$$

whose eigenvectors correspond to values of the expansion coefficients $a_{n, \mathbf{q}}^{(m)}$ which satisfy Eq. (9). The eigenvector corresponding to the smallest eigenvalue is the best optimum.

In practice, it is necessary to select values for the weighting parameters $\beta_{I}$ and $\beta_{\mathcal{V}}$. Nominally, this is because the various terms in the cost functional are dimensionally inequivalent, and their relative magnitudes can differ significantly. For example, the cavity $Q$ and the mode volume are not numbers that can be directly added or compared. The inversion parameters $\beta_{I}$ and $\beta_{\mathcal{V}}$ serve to remedy this problem by mapping both terms into the dimensionless interval $[0,1]$. Therefore, the ideal choices for the weighting parameters are the reciprocals of the optimal mode volume and $Q$.

However, since the optimal values are not known at the beginning of the inversion procedure, it is impossible to know the best choices for $\beta_{I}$ and $\beta_{\mathcal{V}}$ a priori. While one possible solution is to guess values for the inversion parameters that nominally map the terms into the interval $[0,1]$, this is not an algorithmic procedure. A systematic approach to identifying good choices for $\beta_{I}$ and $\beta_{\mathcal{V}}$ is to nest Eq. (24) within a second maximization over the $\beta_{i}$. Although, performing the $\beta_{i}$ optimization calls for repeating the eigenvalue problem, possibly many times, saving the values of the variational matrix elements and simply rescaling them according to the particular choice of the $\beta_{i}$ offers an efficient computational technique for the inversion.

\section{Extracting the defect dielectric}

With the optimal mode coefficients $a_{n, \mathbf{q}}^{(m)}$ known, the final component of the inversion is to extract the defect dielectric $\delta \eta(\mathbf{r})$ from the expansion coefficients. Doing so involves inverting the Maxwell equations, and can be accomplished by substituting Eqs. (10), (11), and (16) into Eq. (1) and solving for the $\delta \eta_{\mathbf{k}}$.

In simplifying the resulting expressions, it is necessary to make use of the orthogonality of the bulk crystal mode functions. It is also helpful to let the mode wave vectors $\mathbf{q}$ run over multiple Brillouin zones. Doing so leads to more manageable equations because the summations are no longer restricted. In the end, the proper expressions can be obtained by folding the equations back into the first Brillouin zone. The details of the derivation are provided in the Appendix, and the result is a linear system of equations,

$$
\sum_{\mathbf{k}} D_{n, \mathbf{q} ; \mathbf{k}}^{(m)} \delta \eta_{\mathbf{k}}=a_{n, \mathbf{q}}^{(m)} \frac{\omega_{m}^{2}-\omega_{n, \mathbf{q}}^{2}}{c^{2}},
$$

where the inversion matrix $\mathbf{D}^{(m)}$ is given by

$$
\begin{aligned}
D_{n, \mathbf{q} ; \mathbf{k}}^{(m)}= & \sum_{n^{\prime}} \sum_{\mathbf{G}, \mathbf{q}^{\prime}} a_{n^{\prime}, \mathbf{q}^{\prime}}^{*} h_{n, \mathbf{q}+\mathbf{G}}^{*} h_{n^{\prime}, \mathbf{q}^{\prime}+\mathbf{G}-\mathbf{k}^{\prime}} \\
& \times(\mathbf{q}+\mathbf{G}) \cdot\left(\mathbf{q}+\mathbf{G}-\mathbf{k}^{\prime}\right) .
\end{aligned}
$$

The matrix is indexed by the bulk modes, labeled by $(n, \mathbf{q})$, and the Fourier coefficients of the defect, $\mathbf{k}$.

An important point to make is that the cavity resonance frequency $\omega_{m}$ enters into Eq. (24) as a parameter. Solving the inversion requires specifying the cavity frequency which can take on any value within the bulk crystal band gap. It should be expected that the best in-plane confinement results from a cavity frequency $\omega_{m}$ deep within the band gap. However, the resulting inverted defect dielectric function is different depending on the choice of the resonance frequency. Moreover, different choices of $\omega_{m}$ might lead to defects that are easier to fabricate than others.

\section{Computational complexity}

Interpreting the computational complexity of the matrix equations in Eq. (25) is aided by considering the $\mathbf{k}$ vectors as a sum of reciprocal lattice vectors and Brillouin zone vectors, $\mathbf{k}=\mathbf{q}+\mathbf{G}$. This shows that the defect is constructed using bulk crystal modes for all wave vectors that lie within the Brillouin zone. Counting vectors in this manner allows the 
dimensions of the inversion matrices to be determined. Since Eq. (1) is Hermitian, the number of bands is equal to the number of reciprocal lattice vectors, $N_{G}=\infty$. Similarly, the number of bulk crystal modes needed to construct a nonperiodic defect is $N_{q}=\infty$. Therefore, the dimension of the square matrix $\mathbf{D}^{(m)}$ is a strictly countable double infinity.

In practice, summations over reciprocal lattice vectors are truncated to a finite number $N_{G}$, which likewise limits the number of bands for each wave vector to $N_{G}$. The truncated bulk mode basis is no longer rigorously complete. However, in practice, the Fourier coefficients in Eq. (10) fall off quickly as $\|\mathbf{G}\|$ increases. Therefore, truncating the basis can be achieved without sacrificing accuracy provided that Fourier expansions remain sufficiently accurate. Retaining the proper dimension of $\mathbf{D}^{(m)}$ requires that the number of defect Fourier wave vectors, $N_{k}$, also be properly chosen. The requirement that $\mathbf{D}^{(m)}$ remain square requires that the number of distinct bulk modes in the Brillouin zone $\mathbf{q}$ (recall that $\mathbf{k}$ $=\mathbf{q}+\mathbf{G})$ be exactly $N_{G}$.

The number of matrix elements in $\mathbf{D}^{(m)}$ scales as $O\left(N_{G}^{2}\right)$. However, the computational complexity of the sums that must be evaluated when computing the matrix elements scale as $O\left(N_{G}^{3}\right)$. Therefore, the overall time complexity for solving the inverse problem is $O\left(N_{G}^{5}\right)$, and can prove to be computationally demanding. In practice, it is beneficial to employ approximate methods for solving the linear system of equations [31], if the calculations are to be performed on a personal computer.

\section{E. Illustration: Symmetric defect}

As a first demonstration, we considered an hexagonal 2D photonic crystal with a radially symmetric defect. The bulk material index of refraction was chosen to be $n_{b}=3.4$, and the hole radius was $r_{h} / a=0.3$ (dimensionless distances, $r / a$, have been adopted due to the invariance of the results with respect to a consistent scaling of the spatial dimensions). The rationale behind these parameters was based on competing factors. It has been shown that large lattice holes lead to out-of-plane loss due to scattering from the edges $[8,22]$. However, the frequency band gap decreases as the hole size is reduced, so $r_{h}$ must not be made too small. $30 \%$ of the lattice constant provides a good compromise.

The dielectric index of refraction, $n_{b}=3.4$, was chosen because it is typical of the semiconductor materials that might be used to incorporate photonic crystals into atom trapping experiments (e.g., $\mathrm{Al}_{0.3} \mathrm{Ga}_{0.7} \mathrm{As}$ ). The essential property of the bulk dielectric material is that it does not absorb light around the atomic transition frequency, for example $852 \mathrm{~nm}$ for Cs.

For the purpose of the calculations, the photonic crystal lattice was truncated to five layers surrounding the center defect at $\mathbf{r}=0$. We found that this number of layers provided a sufficient description of the properties of the photonic crystal without exceeding the computational power of a typical desktop computer. The coefficient optimization was performed by adjusting the parameters, $\left(\beta_{i}\right)$, in $\mathcal{J}$ until the best maximum was achieved. Parameter optimization was performed using a conjugate-gradient search algorithm [32]
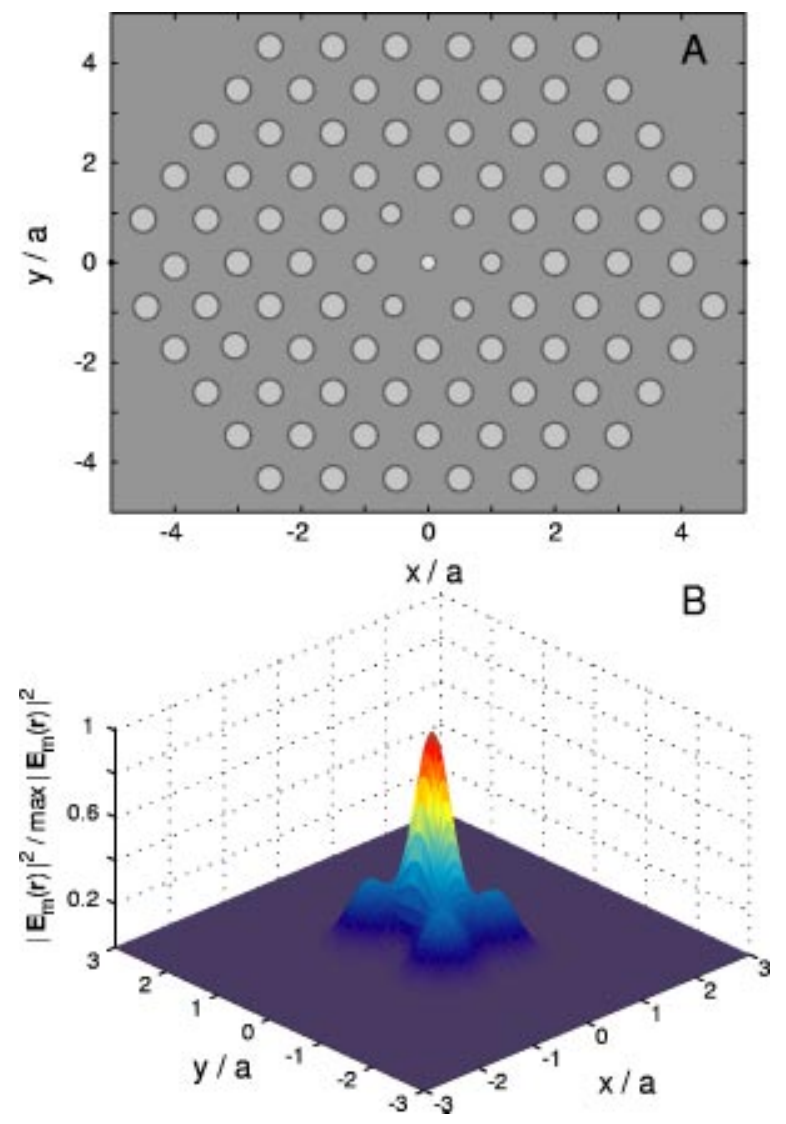

FIG. 1. The photonic crystal (a) and the cavity electric field (b) that result from optimizing the $Q$, mode volume, and peak cavity intensity by solving a two-dimensional inverse problem for a radially symmetric defect. The resulting cavity hole was reduced in radius to $r_{c}=2.1 / a$, where $a$ is the crystal lattice constant, and the neighboring holes were also reduced in size and displaced outward. The most significant feature of the optimized structure is that the index of refraction of the bulk crystal holes was increased to $n_{h}=1.9$.

over the $\beta_{i}$. The search required solving the eigenvalue problem in Eq. (24) for different scaling parameters 28 times.

Solving the linear system of inversion equations in Sec. III D produced the crystal and associated cavity mode shown in Fig. 1. Here, the 2D mode volume was $\sim \lambda^{2} / 4$. The location and size of the photonic crystal holes was determined from a contour plot of the reciprocal dielectric function, see Fig. 2. The contour half way between the minimum and maximum values of $\eta(\mathbf{r})$ was adopted in order to eliminate the small peak oscillations. These oscillations were the result of truncating the Fourier expansions for $\eta_{0}(\mathbf{r})$ and $\delta \eta(\mathbf{r})$.

The photonic crystal shown in Fig. 1(a) has several distinctive features. First, the hole at the location of the cavity is reduced in radius to $r_{c}=0.21 / a$. The nearest-neighbor holes were also reduced in radius, to approximately $r_{h}=0.26 / a$, as well as outwardly displaced from their original locations by $\sim 0.15 / a$. Qualitative arguments for these features, which were also observed by Vuckovic et al., have been proposed [8]. Reducing the size of the defect hole draws a bulk mode from the air band into the photonic band gap. Air-band 


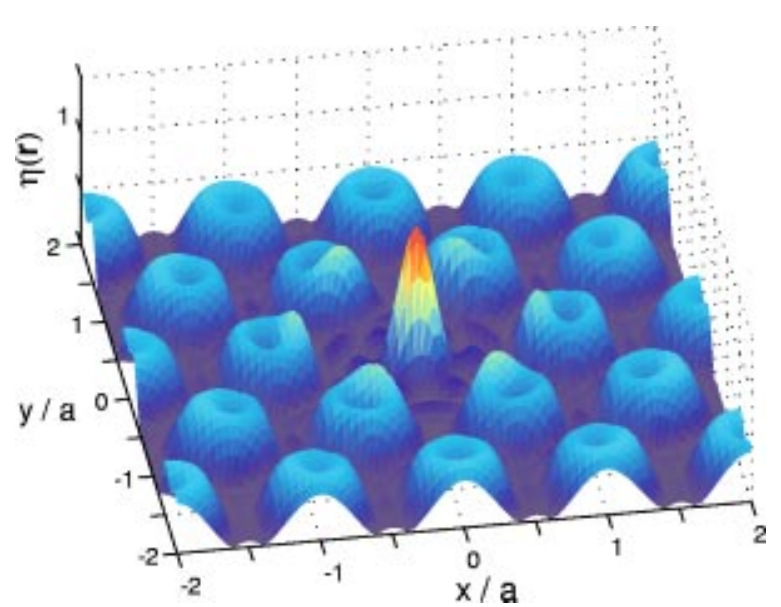

FIG. 2. Extracted reciprocal dielectric function, $\eta(\mathbf{r})=1 / \kappa(\mathbf{r})$, found by solving the inversion problem for a two-dimensional hexagonal lattice with a radially symmetric defect. The most distinctive feature of the mathematically optimal photonic crystal is that the bulk lattice air holes have an index of refraction $n_{h}>1$.

modes are characterized by higher intensities in regions where the index of refraction is lower, such as in the air hole. Therefore, the reduced cavity radius is most likely the result of the design requirement that maximizes the intensity at $\mathbf{r}_{a}$ $=0$.

Decreasing the radii of the neighboring holes and moving them away from the cavity reduces the intensity of the secondary lobes surrounding the main peak in the cavity mode [see Fig. 1(b)]. These lobes, which would normally coincide with air holes in the hexagonal lattice, experience an atypically high index of refraction. The lobe intensities are suppressed because the defect mode, which was pulled from the air band, is low-index seeking. As a result, the cavity mode displays better localization. Therefore, the displaced neighboring holes likely result from the mode volume minimization design requirement.

By far, the most significant feature of the optimized photonic crystal in Fig. 1 is that the index of refraction of the bulk holes is increased to $n_{h} \sim 1.9$. The optimal structure is a bulk photonic crystal whose holes are made of a material other than air. Understanding this result requires analyzing how the cavity mode is constructed from the bulk mode basis functions. The solid lines in Fig. 3 show the dispersion relationship of the optimized crystal (with nonair holes). For reference, the dispersion relationship for a corresponding crystal with air holes $\left(n_{h}=1.0\right)$ is denoted by the broken lines. The points (circles) represent bulk modes, identified by their band index, wave vector, and frequency, which contribute more than $1 \%,\left|a_{n, \mathbf{q}}^{(m)}\right|^{2}>10^{-2}$, to the optimized cavity mode.

As can be seen in Fig. 3, the crystal with $n_{h}=1.9$ contains more bands that lie below the light line. Consequently, more modes, particularly with larger wave vectors and frequencies, contribute to the cavity mode without sacrificing $Q$ (since these modes now lie below the light line). Most likely, the ability to include contributions from more bulk crystal modes allows an increase in the cavity $Q$, while simultaneously decreasing the mode volume.

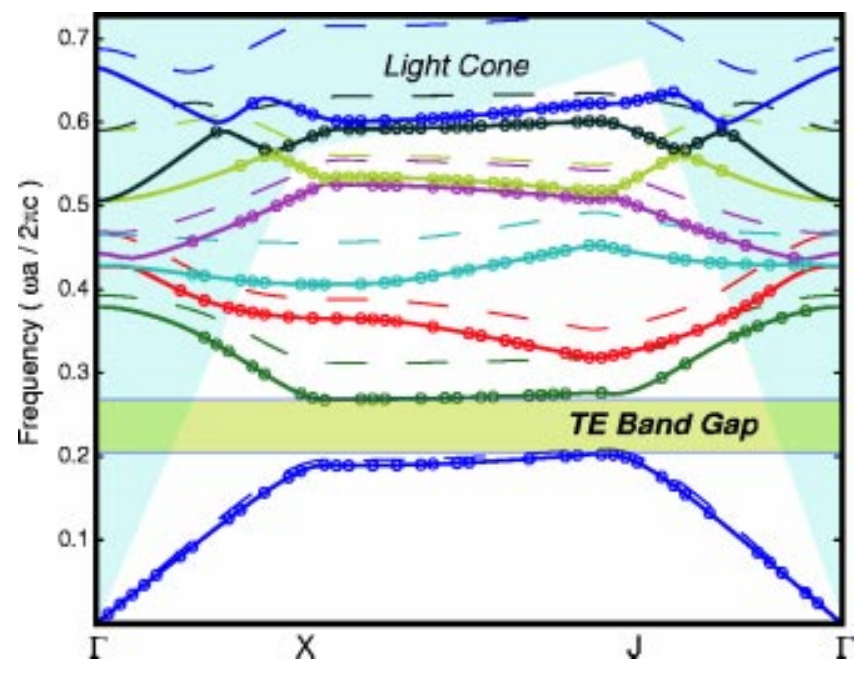

FIG. 3. Dispersion relationship and bulk mode contributions to the optimized cavity mode for a radially symmetric defect. The upper shaded region denotes the free-space light line. The band structure (solid lines) of the optimized photonic crystal is shown and the points indicate bulk modes that contribute more than $1 \%$ to the cavity mode. The dashed lines represent the band structure of a photonic crystal with air holes.

The rationale here is that leaky modes must be excluded by the $Q$ maximization. However, constructing a cavity mode that is well localized, i.e., not periodic, requires a large number of Fourier components, including high frequency modes. Drawing bands out of the light cone provides access to more basis functions and this allows a localized cavity field to be constructed without resorting to leaky modes. Still, the optimization did incorporate several bulk modes from above the light line, particularly from the $J-\Gamma$ line.

We do not attempt to comment on how photonic crystals with nonair holes might be fabricated. However, it is important to consider the major effects of adjusting the hole dielectric. Increasing the hole index of refraction decreases the band gap. However, because the index contrast between the bulk material and the holes is smaller, it should be possible to increase the hole radius without suffering as much vertical scattering from the edges. Of course, increasing the hole size pushes bands back into the light cone and a balance must be found.

\section{F. Illustration: Asymmetric defect}

As a second demonstration, we relaxed the radial symmetry requirement, and considered an arbitrary defect in a twodimensional hexagonal lattice. The same photonic crystal parameters from Sec. III E were adopted: a bulk index of refraction $n_{b}=3.4$, and hole radius $r_{h} / a=0.3$. Again, the mode expansion was constructed using five photonic crystal layers surrounding the defect center to provide a sufficient bulk mode basis expansion without incurring excessive computational expense.

Solving the linear system of inversion equations was performed by nesting the optimization within a conjugategradient search for the best $\beta_{i}$ weighting parameters. The photonic crystal was again constructed by taking the contour 


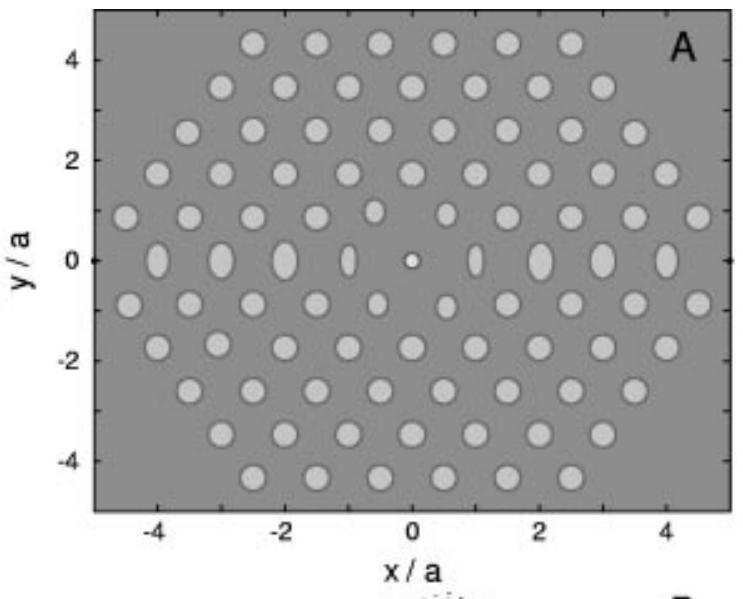

B

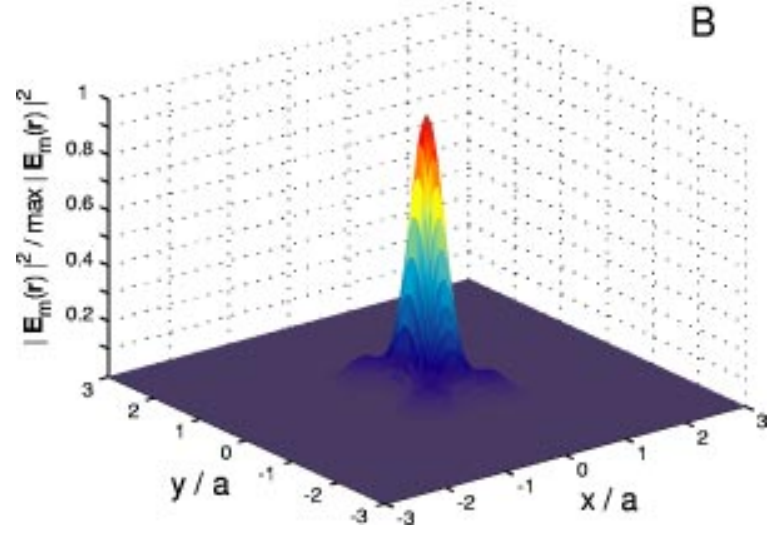

FIG. 4. The photonic crystal (a) and the cavity mode (b) that result from optimizing the $Q$, mode volume, and cavity intensity by solving a two-dimensional inverse problem (radial symmetry not imposed). The cavity hole was reduced in radius to $r_{c}=2.18 / a$, and the neighboring holes were elongated in the vertical direction. The most significant feature of the optimized structure is that the index of refraction of the bulk crystal holes was increased to $n_{h}=1.75$.

level half way between the minimum and the maximum of $\eta(\mathbf{r})$ to eliminate the numerical effects of truncating the Fourier expansions.

The resulting photonic crystal and cavity mode is depicted in Fig. 4. Here, the volume of the $2 \mathrm{D}$ mode was reduced to $\sim \lambda^{2} / 10$. The center photonic crystal hole was reduced in radius to $r_{c}=0.22 / a$ and remained circular. However, the nearest-neighbor holes were deformed mainly in the $\hat{y}$ direction. The minor axes, along $\hat{x}$, of the four holes above and below the cavity were reduced to $r_{h} \sim 0.26 / a$. Their corresponding major axes were simultaneously increased to $0.39 / a$, resulting in the elliptical holes as seen in Fig. 4(a). These four neighboring holes were also radially displaced from their normal hexagonal lattice sites by $0.05 / a$, smaller than what was observed for the symmetric defect.

There was also a deformation of holes lying along the $\hat{x}$ axis. These were elongated in the $\hat{y}$ direction; however, the degree of eccentricity was not constant. The minor axes of the two horizontally neighboring holes decreased to $0.26 / a$, while their major axes increased to $0.31 / a$. The remaining horizonal holes were also vertically stretched, with major axes given by $0.36 / a, 0.31 / a$, and $0.30 / a$ for photonic crystal

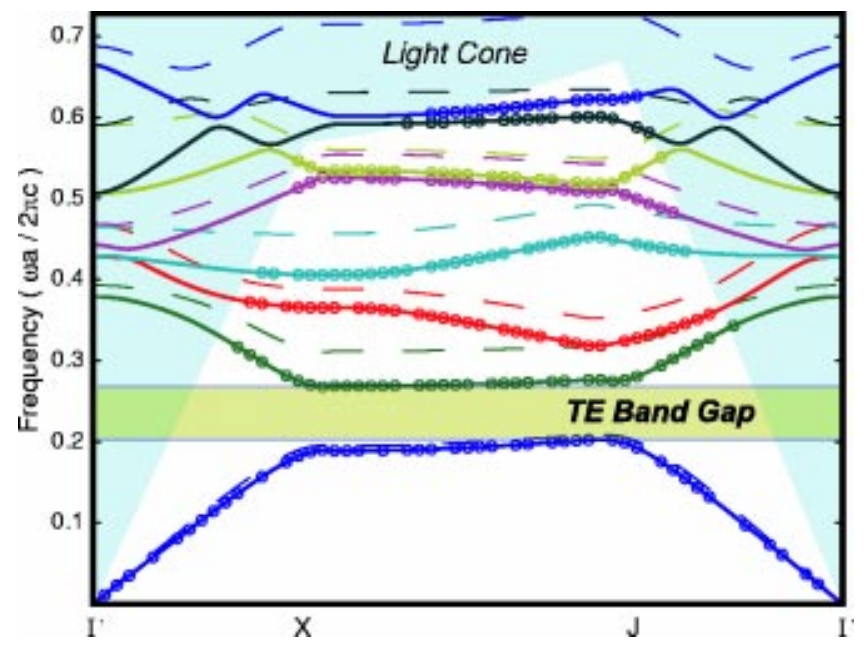

FIG. 5. Dispersion relationship and bulk mode contributions to the optimized cavity mode for a radially symmetric defect. The upper shaded region denotes the free-space light line. The band structure (solid lines) of the optimized photonic crystal is shown and the points indicate bulk modes that contribute more than $1 \%$ to the cavity mode. The broken lines represent the band structure of a photonic crystal with air holes.

layers 3, 4, and 5, respectively. Slight deformations along the $\hat{x}$ direction were observed in the $\hat{y}$-axis holes. However, their deformation was small, with major axes of $0.31 / a$ along the $\hat{x}$ direction and minor axes of $0.29 / a$. No consistent deformation of hole size was observed for any other direction.

The qualitative arguments, suggested by Vuckovic et al. [8], also apply to this structure. Replacing air hole sites with higher-index material suppresses the amplitude of air-band originated modes. But more importantly, the partial elongation of holes along the $\hat{x}$ axis bears significant resemblance to the "fractional edge delocations" suggested by Scherer and co-workers $[8,9]$.

As with the radially symmetric defect, the index of refraction of the bulk holes increased, but only to $n_{h} \sim 1.8$. A similar argument based on pulling higher bands out of the light cone again applies. However, it is not as easy to provide an argument for the elongated holes surrounding the defect and along the $\hat{x}$ and $\hat{y}$ axes. Nonetheless, it can be seen from Fig. 4(b) that better mode localization was achieved by allowing an asymmetric cavity defect.

Figure 3 shows the band diagram for the optimized photonic crystal. The dispersion relationship for bulk holes with $n_{h}=1.8$ is depicted by the solid lines, and the air hole bands are given by the broken lines. The circles represent bulk modes that contribute more than $1 \%, a_{n, \mathbf{q}}^{(m)}>10^{-2}$, to the optimized cavity mode. The same argument used to explain the symmetric defect results can be made here. The crystal with $n_{h}=1.8$ contains more bands below the light line.

A distinctive feature of the optimal asymmetric mode is that it contains fewer contributions from leaky modes (inside the light cone) than for the symmetric case. This can be seen by comparing Figs. 3 and 5. Exactly how the larger $Q$ was achieved for the asymmetric cavity is not clear. However, it is not surprising that constraining the optimization (for ex- 


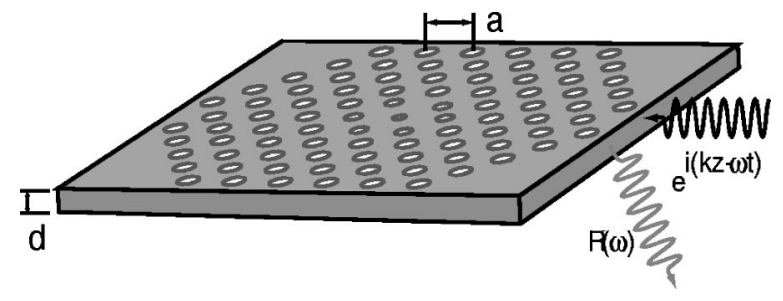

FIG. 6. Schematic of the planar photonic crystal optimized using numerical inversion methods. $a$ is the hexagonal lattice constant, $d$ is the thickness of the slab, and $r_{h}$ is the radius of the bulk crystal holes. The design parameters were obtained by computing the crystal electromagnetic fields produced by plane wave illumination. The cavity $Q$ was obtained by computing the reflection coefficient $R(\omega)$ as a function of incident wave frequency.

ample, by imposing a symmetry restriction) reduces the ability of the inversion to satisfy the design requirements.

In these two-dimensional examples, solving the design problem by inversion resulted in structures that differ from the previous trial and error designs that have been suggested. These new photonic crystal structures, however, might prove difficult to fabricate. As such, they provide an indication of the performance of an ideal, perhaps experimentally impractical, cavity.

\section{NUMERICAL INVERSION RESULTS}

As a final example of inverting a photonic crystal defect, we considered a hexagonal structure with finite depth to enable a direct calculation of the cavity $Q$. Additionally, we constrained the index of refraction of the crystal holes to $n_{h}=1$. In this demonstration, the aim was to identify an optimal planar photonic structure without requiring a more complicated fabrication.

For the planar structure, it was necessary to abandon an analytical solution and perform the inversion optimization numerically. In some respects, the numerical design problem was much simpler than the analytic case - it was possible to maximize the inversion cost functional $\mathcal{J}$, without the need for a specific mathematical analysis. Instead, the spatial dependence of the dielectric function was parametrized. Then the inverse problem was solved by optimizing $\mathcal{J}[\eta(\mathbf{r})]$ over the parameter space using a genetic algorithm.

However, numerical methods introduce several new challenges. For example, since the inversion cost functional involved a large number of parameters, the resulting multidimensional optimization was complicated by the existence of local minima. Additionally, numerically integrating the Maxwell equations is computationally expensive. For instance, a single mode volume and cavity $Q$ calculation can require several minutes of computer time.

\section{Planar photonic crystal}

We considered an eight layer, two-dimensional hexagonal lattice slab, as depicted in Fig. 6. Based on the previous arguments (c.f., Sec. III E), the hole radius was chosen to be $r_{h} / a=0.3$ with a bulk material index of refraction $n_{b}=3.4$. The slab thickness was taken to be $d / a=3 / 4$, in order to

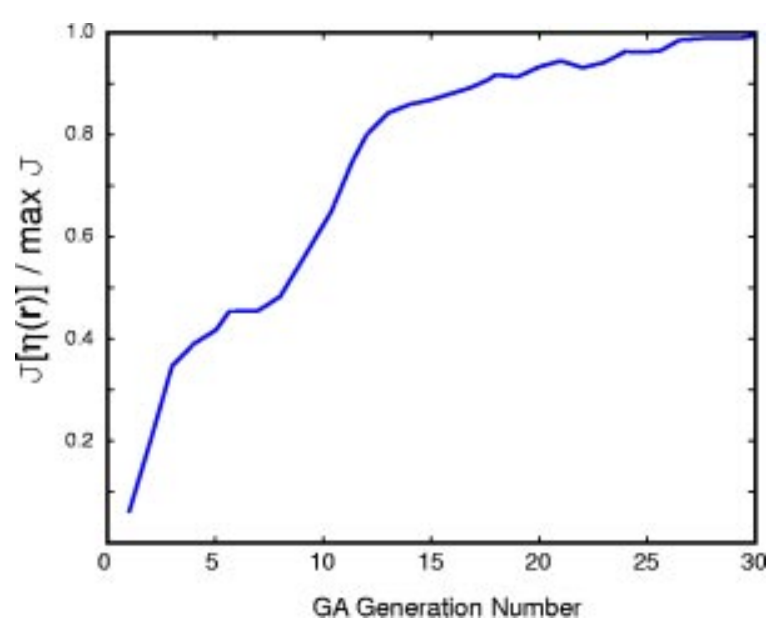

FIG. 7. Optimization progress as a function of genetic algorithm generation number for the numerical inversion involving a planar photonic crystal.

prevent multimode behavior [7]. Parametrizing the dielectric function was accomplished by treating the positions and major and minor axes of the lattice holes as optimization variables. Specifically, the cavity hole, the holes surrounding the defect, and the $\hat{x}$-axis holes were considered. In all, there were 52 optimization variables, 4 for 13 different lattice sites.

The Maxwell equations were solved by employing the transfer matrix method of Pendry and Bell [33]. This technique provided a means for computing the stationary states of the electromagnetic field for a crystal illuminated on one edge by a plane wave (refer to Fig. 6). The spatial dependence of the electromagnetic fields, as well as the crystal transmission and reflection coefficients were calculated using a first-order finite difference solution to Maxwell's equations. The integration mesh used to compute the wave fields extended five layers above and below the surface of the slab, and absorbing boundary conditions were imposed at the top and bottom of the integration cell.

The mode volume was computed by integrating the square magnitude of the electric field over the interior of the photonic crystal. The volume integration was performed using a three-dimensional Simpson's rule quadrature, and the result was scaled by the field maximum, according to Eq. (3). The cavity $Q$ was directly computed by scanning the reflection coefficient over the frequency band gap. The full width at half maximum of the reflection line shape was then used to determine the cavity $Q$.

The inversion cost functional was optimized by employing a genetic algorithm [34] (GA) to maximize $\mathcal{J}[\eta(\mathbf{r})]$. A GA was chosen because, although not terribly efficient, genetic algorithms provide good exploration to exploitation in multidimensional searches. As such, they are generally successful at avoiding local minima on a complex optimization surface. However, good exploration does not come without cost. Twenty-seven GA generations (iterations of the algorithm) were required to optimize $\mathcal{J}$, as can be seen Fig. 7. With a population size of 10 , a steady-state propagation routine, a mutation rate of $15 \%$ and a crossover rate of $85 \%$, the 

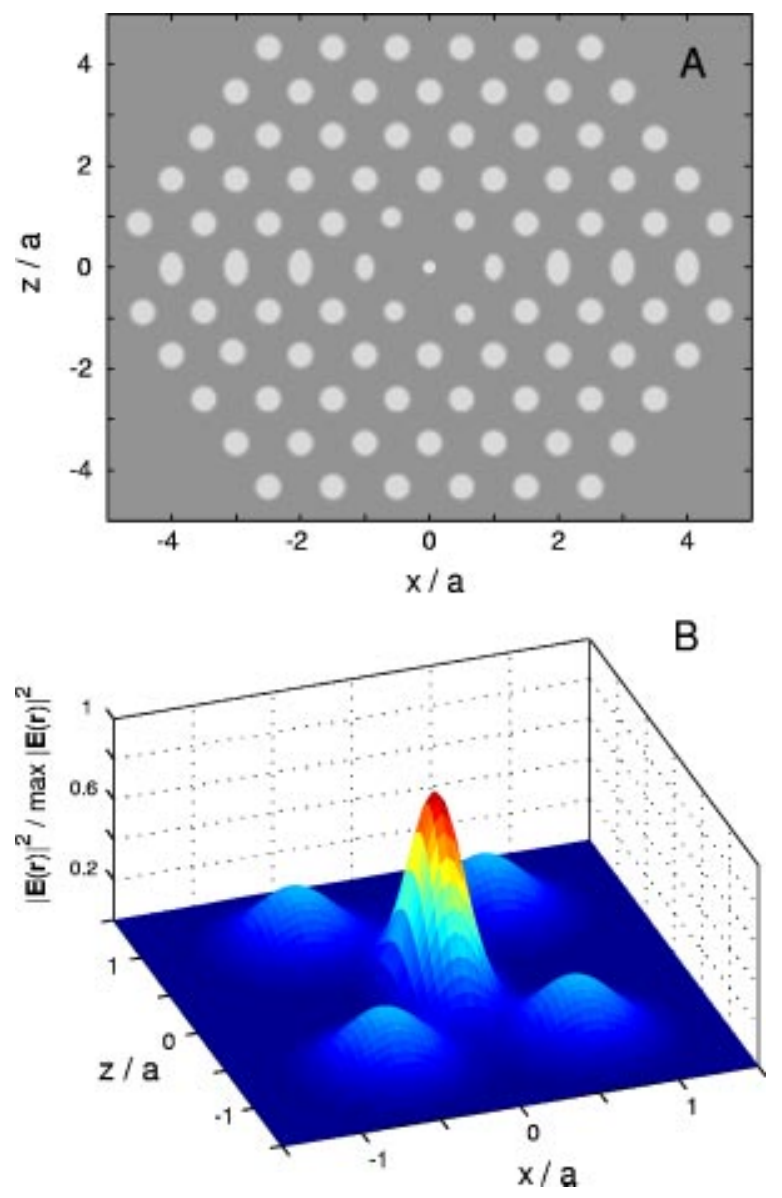

FIG. 8. The photonic crystal (a) and the cavity electric field (b) that result from optimizing the $Q$, mode volume, and peak cavity intensity by performing a numerical inversion for a planar photonic crystal.

full optimization required $24.5 \mathrm{~h}$ of central processing unit time on an Intel PIII 1.2-GHz desktop machine.

The resulting photonic crystal and the optimized cavity mode are shown in Fig. 8. As can be seen, a similar structure to the two-dimensional optimization was obtained. The cavity defect hole was reduced in radius to $0.21 / a$ and the holes surrounding the defect were also decreased in size. The holes along the $\hat{x}$ axis were also elongated in the $\hat{z}$ direction, which again demonstrated the remarkable similarity to the fractional edge delocations suggested by Scherer and co-workers $[8,22]$.

The mode volume of the field in Fig. 8 was found to be $\mathcal{V}_{m} \sim \lambda^{3} / 3$ and the cavity $Q$ was $\sim 1.1 \times 10^{5}$. These quantities surpass those of the best PBG cavity designs to date.

\section{CONCLUSION}

We demonstrated that inversion methods provide a powerful technique for designing photonic crystals for cavity QED experiments. Both an analytical solution for a twodimensional crystal and numerical results for a planar (finite thickness) crystal were presented. In both cases, the design objectives, namely high cavity $Q$ and small mode volume, were achieved. The analytical analysis on 2D structures of- fered the advantage that no specific parametrization of the dielectric function was necessary, and the structure was free to explore full variations consistent with the imposed hexagonal symmetry. The numerical illustrations, while they required a specific parametrization of the dielectric function, demonstrated the success of inversion techniques for structures that can be tested in the laboratory.

For two-dimensional crystals, it was shown that contributions to the cavity mode from leaky bulk modes could be minimized without delocalizing the field. Here, inversion produced design alternatives not previously suggested by trial and error, or intuitive design. The optimal cavity defect for both the radially symmetric and asymmetric defect optimizations called for a lattice of holes with an index of refraction greater than 1. An explanation for this inversion result, i.e., bulk crystal holes composed of a material other than air, was suggested. Increasing the index of refraction of the lattice holes pulls more of the band diagram out of the light cone. Consequently, less of the higher frequency bulk modes are leaky. It was argued that a better localized cavity field can be constructed by incorporating these higher frequency basis functions.

A point from the analytic inversion that deserves some attention is the choice of cavity resonance frequency $\omega_{m}$. It can be seen in Eq. (25) that $\omega_{m}$ enters the inversion as a parameter which must be specified. A thorough investigation of the inversion outcome as a function of where $\omega_{m}$ lies within the band gap was not performed here because it would require repeating the full inversion for many different choices of $\omega_{m}$ in order to fully characterize the relationship. The computational expense of such an analysis is prohibitive. Further investigations may attempt to address this point.

The inverse problem design approach was also applied to a planar crystal in order to treat a more realistic structure. However, this required that numerical methods for integrating the Maxwell equations as well as optimizing the inversion cost function be adopted. It was demonstrated that a crystal with low mode volume and high $Q$ could be achieved. In order to minimize the effects of local minima in the inversion optimization, a genetic algorithm was adopted, despite its computational expense.

For the planar photonic crystal inversion, the dielectric constant of the bulk lattice holes was constrained to $n_{h}=1$. This provided an example of restricting the inversion optimization because of fabrication considerations. Although it may be possible to manufacture the inversion designs from Sec. III, i.e., bulk lattice holes with $n_{h}>1$, it is also important to identify the best possible structure which canbe fabricated using the currently available techniques.

An aspect of the design process that was not considered in this paper was robustness. Ideally, the photonic crystal would be insensitive to slight variations in its structure, as might result from small fabrication errors, temperature fluctuations, etc. Including robustness into design optimization problems would add additional constraints into the optimization process, however, algorithmic approaches to finding robust optima are known $[35,36]$. In addition to investigating robustness, we plan to explore the use of optimization methods to design PBG structures with convenient geometric features, 
such as enlarged holes for atom trapping.

It was shown that the mathematical analysis tools utilized here simultaneously optimize multiple, complimentary design requirements. Achieving similar outcomes via trial and error methods is generally a formidable task. Therefore, an algorithmic approach to design problems with multiple objectives, such as with photonic band gap materials, will likely only be possible via an algorithmic approach.

\section{ACKNOWLEDGMENT}

This work was supported by the DoD Multidisciplinary University Research Initiative (MURI) program administered by the Army Research Office under Grant No. DAAD19-00$1-0374$

\section{APPENDIX: 2D INVERSION EQUATIONS}

In this appendix, we describe in greater detail how to extract the defect dielectric function from the optimized expansion coefficients (refer to Sec. III D). The general procedure involves substituting the normal mode expansion (11) and the Fourier expansions (10) and (16), into the Maxwell equation (1). The resulting inversion equation (24) results from employing the orthogonality of the bulk modes.

In doing the algebra, it is more convenient to work a bulk mode expansion that includes wave vectors from all of $k$ space, i.e., by incorporating multiple Brillouin zones,

$$
\mathbf{H}_{m}(\mathbf{r})=\frac{1}{N} \sum_{n, \mathbf{k}} a_{n, \mathbf{k}}^{(m)} H_{n, \mathbf{k}}(\mathbf{r})
$$

where $N$ is the number of Brillouin zones (reciprocal lattice vectors). The orthogonality relationships are now give by

$$
\int_{V_{N}} \mathbf{H}_{n^{\prime}, \mathbf{k}^{\prime}}^{*}(\mathbf{r}) \mathbf{H}_{n, \mathbf{k}}(\mathbf{r}) d \mathbf{r}=\delta_{n, n^{\prime}} \sum_{\mathbf{G}} \delta_{\mathbf{k}^{\prime}, \mathbf{k}+\mathbf{G}},
$$

where the integration is over the $N$ associated Wigner-Seitz cells.
Substituting both the bulk crystal and defect dielectric Fourier expansions into the Maxwell curl equation,

$$
\boldsymbol{\nabla} \times \eta_{0}(\mathbf{r}) \boldsymbol{\nabla} \times \mathbf{H}_{m}(\mathbf{r})+\boldsymbol{\nabla} \times \delta \eta(\mathbf{r}) \boldsymbol{\nabla} \times \mathbf{H}_{m}(\mathbf{r})=\frac{\omega_{m}^{2}}{c^{2}} \mathbf{H}_{m}(\mathbf{r}),
$$

left-multiplying by $\mathbf{H}_{n^{\prime \prime}, \mathbf{q}^{\prime \prime}}$ and integrating leads to

$$
\begin{aligned}
\frac{1}{N} \sum_{n, \mathbf{k}} & a_{n, \mathbf{k}}^{(m)} \frac{\omega_{n, \mathbf{k}}^{2}-\omega_{m}^{2}}{c^{2}} \int_{V} \mathbf{H}_{n^{\prime \prime}, \mathbf{k}^{\prime \prime}}^{*}(\mathbf{r}) \mathbf{H}_{n, \mathbf{k}}(\mathbf{r}) d \mathbf{r} \\
= & \frac{1}{N} \sum_{\mathbf{k}} \sum_{n, \mathbf{k}} \sum_{\mathbf{G}, \mathbf{G}^{\prime \prime}} a_{n, \mathbf{k}}^{(m)} \delta \eta_{\mathbf{k}} h_{n^{\prime \prime}, \mathbf{k}^{\prime \prime}+\mathbf{G}^{\prime \prime}}^{*} h_{n, \mathbf{k}+\mathbf{G}} \\
& \times\left(\mathbf{k}^{\prime \prime}+\mathbf{G}^{\prime \prime}\right) \cdot\left(\mathbf{k}^{\prime \prime}+\mathbf{G}^{\prime \prime}-\mathbf{k}^{\prime}\right) \int_{V} e^{i\left(\mathbf{k}+\mathbf{G}+\mathbf{k}^{\prime}-\mathbf{k}^{\prime \prime}-\mathbf{G}^{\prime \prime}\right) \cdot \mathbf{r}} d \mathbf{r},
\end{aligned}
$$

where, again, the integrations are over the $N$ unit cells. Applying the orthogonality of the bulk modes leads to a set of equations that still run over all wave vectors,

$$
\begin{aligned}
\frac{1}{N} \sum_{n, \mathbf{k}} & \sum_{\mathbf{k}^{\prime}} \sum_{\mathbf{G}^{\prime \prime}} a_{n, \mathbf{k}}^{(m)} \delta \eta_{\mathbf{k}^{\prime}} h_{n^{\prime \prime}, \mathbf{k}^{\prime \prime}+\mathbf{G}^{\prime \prime}}^{*} h_{n, \mathbf{G}^{\prime \prime}-\mathbf{k}^{\prime}} \\
& \times\left(\mathbf{k}^{\prime \prime}+\mathbf{G}^{\prime \prime}\right) \cdot\left(\mathbf{k}^{\prime \prime}+\mathbf{G}^{\prime \prime}-\mathbf{k}^{\prime}\right) \\
= & \frac{-1}{N} a_{n^{\prime \prime}, \mathbf{k}^{\prime \prime}}^{(m)} \frac{\omega_{n^{\prime \prime}, \mathbf{k}^{\prime \prime}}^{2}-\omega_{m}^{2}}{c^{2}} .
\end{aligned}
$$

Then, the summations can be folded back into the first Brillouin zone by using the identity

$$
\sum_{n, \mathbf{k}} a_{n, \mathbf{k}}^{(m)}=N \sum_{n, \mathbf{q}} a_{n, \mathbf{q}}^{(m)}
$$

and the indices can be renamed to produce Eq. (24).
[1] A. Tihkonov and V. Arsenin, Solutions of Ill-posed Problems (Winston/Wiley, Washington, 1977).

[2] Quantum Inversion Theory and Applications, edited by $\mathrm{H}$. Geramb (Springer-Verlag, New York, 1994).

[3] J. Joannopoulos, R. Meade, and J. Winn, Photonic Crystals (Princeton University Press, Princeton, 1995).

[4] E. Yablonovitch, T. Gmitter, and K. Leung, Phys. Rev. Lett. 67, 2295 (1991).

[5] A. Scherer, T. Doll, and E. Yablonovitch, Lightwave Technol. 17, 11 (1999).

[6] M. Plihal and A.A. Maradudin, Phys. Rev. B 44, 8565 (1991).

[7] M. Loncar, D. Dedeljkovic, T. Doll, J. Vuckovic, A. Scherer, and T. Pearsall, Appl. Phys. Lett. 77, 1937 (2000).

[8] J. Vuckovic, M. Loncar, H. Mabuchi, and A. Scherer, Phys. Rev. E 65, 016608 (2001).

[9] J. Vuckovic, M. Loncar, H. Mabuchi, and A. Scherer, Proceedings of LEOS, 2000, (2000).
[10] H. Mabuchi, M. Armen, B. Lev, M. Loncar, J. Vuckovic, H.J. Kimble, J. Preskill, M. Roukes, and A. Scherer, Quantum Inf. Comput. 1, 7 (2001), special issue on implementation of quantum computation.

[11] M. Woldeyohannes and S. John, Phys. Rev. A 60, 5046 (1999).

[12] T. Yoshie, A. Scherer, H. Chen, D. Huffaker, and D. Deppe, Appl. Phys. Lett. 79, 114 (2001).

[13] J.D. Weinstein and K.G. Libbrecht, Phys. Rev. A 52, 4004 (1995).

[14] E. Miyai and K. Sakoda, Opt. Lett. 26, 740 (2001).

[15] C. Smith, T. Krauss, H. Benisty, M. Rattier, C. Weisbuch, U. Eosterle, and R. Houdre, J. Opt. Soc. Am. B 17, 2043 (2000).

[16] S. Noda, A. Chutinan, and M. Imada, Nature (London) 407, 608 (2000).

[17] P. Villeneuve, S. Fan, S. Johnson, and J. Joannopoulos, IEEE Proc.: Optoelectron. 145, 384 (1998). 
[18] O. Painter, R. Lee, A. Scherer, A. Yariv, J. O’Brien, P. Dapkis, and I. Kim, Science 284, 1819 (1999).

[19] S. Johnson, S. Fan, A. Mekis, and J. Joannopoulos, Appl. Phys. Lett. 78, 3388 (2001).

[20] N. Kawai, K. Inoue, N. Carlsson, K. Ikeda, Y. Sugimoto, K. Asakawa, and T. Takemori, Phys. Rev. Lett. 86, 2289 (2001).

[21] O. Painter, J. Vuckovic, and A. Sherer, J. Opt. Soc. Am. B 16, 275 (1999).

[22] J. Vuckovic, M. Loncar, H. Mabuchi, and A. Scherer, IEEE J. Quantum Electron. (to be published).

[23] A. Scherer, O. Painter, B. D'Urso, R. Lee, and A. Yariv, J. Vac. Sci. Technol. B 16, 3906 (1998).

[24] Bold text in this paper is used to represent vector quantities.

[25] Cavity Quantum Electrodynamics, 1st ed., edited by P.R. Berman, Advances in Atomic, Molecular and Optical Physics Vol. 2 (Academic Press, New York, 1994).

[26] In some cases, the polarization may be position dependent, leading to $\mathbf{H}_{j}^{0}=\mathbf{H}_{j}^{0}(r)$.

[27] In this paper, the index $j$ refers to any mode supported by the photonic crystal, while $m$ is the cavity mode

[28] M. Plihal, A. Shambrook, and A.A. Maradudin, Opt. Commun. 80, 199 (1991).
[29] N.W. Ashcroft and W.D. Mermin, Solid State Physics (Saunders College Publishing, Toronto, 1976).

[30] In this paper, $\mathbf{G}$ always represents a reciprocal lattice vector, $\mathbf{q}$ refers to a wave vector confined to the first Brillouin zone, and $\mathbf{k}$ refers to any wave vector that is not necessarily on the reciprocal lattice or in the Brillouin zone.

[31] G.H. Golub and C.F. van Loan, Matrix Computations, Johns Hopkins Series in the Mathematical Sciences Vol. 3 (Johns Hopkins University Press, Baltimore, 1983).

[32] W. Press, S. Teukolsky, W. Vetterling, and B. Flannery, Numerical Methods in C (Cambridge University Press, New York, 1992).

[33] J. Pendry and P. Bell, in Photonic Band Gap Materials, Vol. 315 of NATO Advanced Studies Institute, Series E: Applied Sciences (Kluwer, Dordrecht, 1996), p. 203.

[34] D. Goldberg, Genetic Algorithms in Search, Optimization, and Machine Learning (Addison-Wesley, Reading, MA, 1989).

[35] G.E. Dullerud and F. Paganini, A Course in Robust Control Theory: A Convex Approach, Texts in Applied Mathematics (Springer-Verlag, New York, 2000) Vol. 36.

[36] J. Geremia, W. Zhu, and H. Rabitz, J. Chem. Phys. 113, 10841 (2000). 